Article

\title{
Design and Performance Analysis of Capacitive Micromachined Ultrasonic Transducer Linear Array
}

\section{Hongliang Wang ${ }^{1,2, *}$, Xiangjun Wang ${ }^{1}$, Changde $\mathrm{He}^{2}$ and Chenyang Xue ${ }^{2}$}

1 State Key Laboratory of Precision Measuring Technology and Instruments, MOEMS Education Ministry Key Laboratory, Tianjin University, Tianjin 300072, China; E-Mail: xdocuxjw@vip.163.com

2 National Key Laboratory for Electronic Measurement Technology, Key Laboratory of Instrumentation Science \& Dynamic Measurement (North University of China), Ministry of Education, North University of China, Taiyuan 030051, China;

E-Mails: hechangde@foxmail.com (C.H.); xuechenyang@nuc.edu.cn (C.X.)

* Author to whom correspondence should be addressed; E-Mail: nucwanghongliang@163.com; Tel.: +86-351-355-7385.

Received: 2 May 2014; in revised form: 18 June 2014 / Accepted: 26 June 2014 /

Published: 3 July 2014

\begin{abstract}
An ultrasonic transducer is a key component to achieve ultrasonic imaging. This paper designs a new type of Microelectromechanical Systems (MEMS) based capacitive ultrasonic transducer and a linear array based on the transducer. Through directivity analysis, it can be found that its directivity is weak due to the small size of the designed transducer, but the directivity of the designed linear array is very strong. In order to further suppress the sidelobe interference and improve the resolution of the imaging system and imaging quality, the Dolph-Chebyshev weighting method and the Taylor weighting method are used to process $-40 \mathrm{~dB}$ sidelobe suppression, and satisfactory results are obtained, which can meet actual requirements.
\end{abstract}

Keywords: capacitive ultrasonic transducer; linear array; beam directivity; sidelobe suppression 


\section{Introduction}

Ultrasonic transducer is an important component to realize the conversion between acoustic energy and electrical energy, which is widely used in medical imaging, non-destructive evaluation, flow measurement, ultrasonic cleaning, distance measurement, etc. Meanwhile, the technology of using ultrasonic transducer to implement underwater acoustic imaging can be used in underwater topography detection, undersea resources survey, underwater operation monitoring, underwater archaeology, etc. Micromachined ultrasonic transducer (MUT) is a new form of ultrasonic transducer made by the technology of microelectronics and micromachining processing. From a processing technique perspective, MEMS technology, compared with thick film processing technology of the integrated piezoelectric diaphragm, controls the accuracy in micrometers $(\mu \mathrm{m})$ magnitude, and it can effectively reduce error of the device structure size processing, so as to improve the consistency of device performance between the arrays. Meanwhile, in terms of the imaging principle, MEMS technology utilizes the silicon piezoresistivity or capacitance change to achieve the detection of acoustic signals, and it can meet the imaging requirements of devices in mall size, so that imaging resolution can be improved. Currently, MEMS technology has been developed internationally to produce an ultrasonic imaging sonar system. With the improvement of MUT design and micromachining technology, MUT has become a promising alternative to traditional ultrasonic transducer and is one of important research directions of the ultrasonic transducer. MUT research mainly includes piezoelectric MUT (PMUT) and capacitive MUT (CMUT). PMUT and CMUT have their own advantages, which are complementary and develop respectively [1].

Currently, PMUT is widely used as an ultrasonic transducer, which is generally made of a vibration membrane and piezoelectric thin film with the upper and lower electrodes. When the transducer is operating in the receiving model, deformation of vibration membrane occurs under the acoustic pressure. At the same time, due to deformation, a piezoelectric thin film on the vibration membrane will produce a corresponding charge, so that acoustic signals can be converted into electrical signals to realize the receiving function. When the transducer is applied to the transmitting model, voltage is applied in the two electrodes of the piezoelectric layer. Deformation of the piezoelectric layer occurs due to the inverse piezoelectric effect, producing the deformation of whole vibration membrane. When the voltage is alternating voltage, vibration of the vibration membrane will occur in the corresponding form, so that acoustic energy can be radiated outward, achieving the conversion from electrical energy to acoustic energy. In recent years, many scholars have carried out a lot of research work about PMUT, mainly including the selection of vibration membrane of the transducer and the piezoelectric layer materials, the improvement of the structure of the transducer and its technological process, etc., and have developed some ultrasonic transducers. For example, the Institute of Acoustics Chinese Academy of Sciences designed a kind of micro ultrasonic transducer based on $\mathrm{ZnO}$ thin films [2], of which the thickness of the $\mathrm{ZnO}$ thin film is $0.5 \mu \mathrm{m}$ and the thickness of the vibration basement membrane is $1 \mu \mathrm{m}$. The shape of the transducer is square with $2 \mathrm{~mm}$ side length and its resonance frequency is $43.7 \mathrm{kHz}$. Meanwhile, Tsinghua University developed a micro ultrasonic transducer for handwriting input system based on PZT thin film [3,4], of which the sensitivity can be changed by adjusting the electrode parameters and the thickness of PZT thin film is $1.8 \mu \mathrm{m}$, the vibration membrane thickness is $1 \mu \mathrm{m}$. The transducer is square with $1 \mathrm{~mm}$ side length and its working frequency is $44 \mathrm{kHz}$. The Federal Institute of 
Technology in Sweden studied a piezoelectric ultrasonic transducer based on PZT thin film [5,6], of which the thickness of the PZT thin film is $1 \mu \mathrm{m}$ and the thickness of the vibration membrane is $10 \mu \mathrm{m}$. The transducer is circular with $150 \mu \mathrm{m}$ radius and the resonance frequency is $1.2 \mathrm{MHz}$. Nanyang Technological University in Singapore also designed a piezoelectric ultrasonic transducer based on PZT thin film [7,8], of which the thickness of the PZT thin film is $7 \mu \mathrm{m}$ and the thickness of the vibration membrane is $1.8 \mu \mathrm{m}$. The transducer is square with $2 \mathrm{~mm}$ side length and the working frequency is $41 \mathrm{kHz}$.

The impedance of traditional PMUT is quite high and ranges from 10-30 MRayl, close to the acoustic impedance of solid medium, which can decrease transmission loss of acoustic wave in the solid. Therefore, the desired test results can be obtained when it is used to detect solid medium. However, compared with PMUT impedance, the acoustic impedance of air and fluid medium is much smaller and both of them cannot match each other. As a result, satisfactory results cannot be obtained by PMUT in air and fluid medium, and the preparation of surface matching layer is usually required to overcome this defect, which can lead to complex process and increasing cost. In addition, traditional PMUT has a lot defects such as short detection distance, small operating temperature range, so there are many constraints in its development and application. In recent years, the capacitive micromachined ultrasonic transducer (CMUT) based on MEMS has gradually become a hot research topic. Ultrasonic emission source of CMUT is a pretty thin film, which makes its impedance lower and can match well the acoustic impedance of air and fluid medium. What is more, the defects of traditional PMUT impedance mismatch can be improved without adding the surface matching layer. CMUT based on MEMS is of small size, low noise and wide operating temperature range; therefore, pre-driver circuit, preamplifier and signal processing circuits can be integrated on a same silicon wafer, which can be widely used in ultrasonic nondestructive detection and medical imaging and many other fields [9-14]. In 1989, researchers in the NEC Corporation proposed an electrostatic ultrasonic transducer in an etched cavity of silicon substrate, which was already a prototype of micro capacitive ultrasonic transducer [15]. In 1994, Khuri-Yakub, research group of Stanford, E.L. Ginzton Laboratory (Stanford, CA, USA) used surface micromachined technology to produce a micro capacitive ultrasonic transducer [16], and in 1999, the same team developed air-coupled nondestructive evaluation using micromachined ultrasonic transducers [17]. In 2002, a linear CMUT array was developed by Oralkan et al. from Stanford University, and then the team developed a 2D CMUT array which could implement 3D ultrasonic imaging [18-28], and conducted preliminary simulation experiments on imaging. In 2006, Caronti et al. developed a one-dimensional array of ultrasonic transducers and detectors used in medical ultrasonic imaging [29]. Recently, some institutes in China have researched CMUT and made achievements. Tianjin University launched a research program, which used a CMUT array as the imaging planar array through MEMS [30]. Tian et al. from the Institute of Acoustics Chinese Academy of Sciences (Beijing, China) developed a micro capacitive microphone of circular structure [31]. Hao et al. from the Institute of Acoustics Chinese Academy of Sciences analyzed the CMUT resonant frequency through the finite element method [32]. Miao et al. from North University of China (Taiyuan, China) studied the method of designing MEMS capacitive ultrasonic transducer based on silicon wafer bonding process [33]. However, all these designs and research mentioned above did not involve actual manufacture of the device. Therefore, carrying out research into CMUT design, simulation, manufacture and test helps to promote the development of CMUT and improvement of related technologies, which is of great research value and practical significance. 
In view of CMUT and its array research, this paper designs a type of CMUT composed of multiple small units, and linear CMUT array is designed by taking it as array element. As for this linear array, this paper analyzes the beam directivity, and studies methods of reducing its beam sidelobe.

\section{MEMS Capacitive Ultrasonic Transducer and Linear Array Design}

Based on an integrated vibration membrane structure, this paper designs a kind of CMUT consisting of $25 \times 2$ micro vibration units. Each unit consists of a substrate, insulating layer, vacuum cavity upper electrode, edge support and so on, and its structure is shown in Figure 1.

Figure 1. Capacitive micromachined ultrasonic transducer (CMUT) structure of micro vibration unit: (a) profile view of CMUT micro vibration unit; (b) top view of CMUT micro vibration unit.

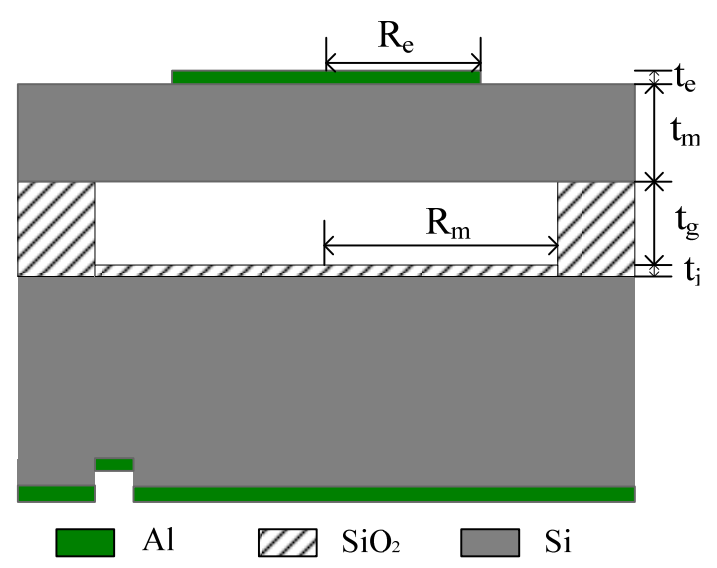

(a)

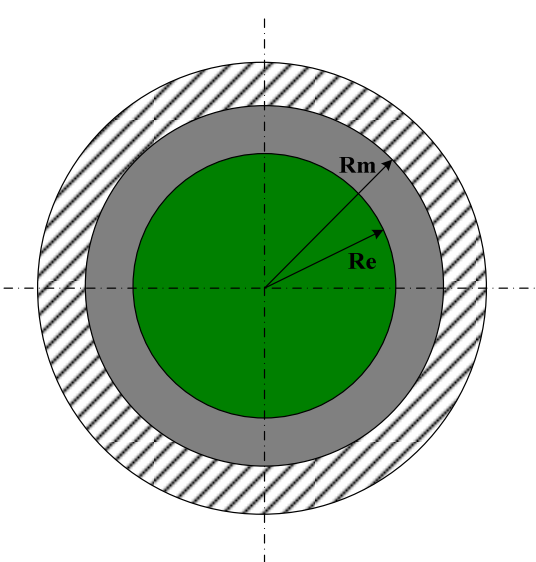

(b)

In Figure $1, t_{\mathrm{e}}$ is the thickness of the upper electrode, $t_{\mathrm{m}}$ is the thickness of the film, $t_{\mathrm{g}}$ is the thickness of the cavity, $t_{\mathrm{i}}$ is the thickness of insulating layer, $R_{\mathrm{m}}$ is the radius of the vibrating film, $R_{\mathrm{e}}$ is the radius of the upper electrode. The silicon wafer is taken as the substrate, and a thin metal layer is deposited as the lower electrode of vibration unit on the silicon substrate. The insulating layer plays a protective role during etching manufacture, and can also guarantee the electrical insulation characteristics between the upper and lower electrodes, which prevents short circuit from occurring caused by accidental engagement of the upper and lower electrodes. The edge support plays a supporting role on the vibration membrane, and helps form a capacitor. On the upper surface of the vibration membrane, a thin layer of metal deposition is taken as the upper electrode of the transducer. Voltage is applied between the upper electrode and the lower electrode to form micro capacitive ultrasonic unit with vibration membrane. Each CMUT Consists of $25 \times 2$ micro vibration units, and the diameter of each vibration unit is $290 \mu \mathrm{m}$, both the vertical distance and horizontal distance between the two vibration units are $30 \mu \mathrm{m}$, so the length of CMUT is $7970 \mu \mathrm{m}$, and the width is $610 \mu \mathrm{m}$.

In order to improve imaging resolution and imaging quality in an ultrasonic imaging system, one-dimensional or two-dimensional linear array ultrasonic transducer is often used to implement the ultrasonic imaging. This study makes 64 CMUT array elements integrated on a silicon wafer by MEMS technology, which compose a CMUT linear array, such CMUT called array element. In this paper, the center frequency of the linear array is $1 \mathrm{MHz}$, and acoustic velocity in water is $1540 \mathrm{~m} \cdot \mathrm{s}^{-1}$. 
Corresponding to the center frequency of the array, the wavelength $\lambda$ is $1540 / 1,000,000=1540 \mu \mathrm{m}$, and the distance between two array elements' center is half wavelength $\lambda / 2$, which is $770 \mu \mathrm{m}$. Sixty four CMUT linear array structure is shown in Figure 2.

Figure 2. Sixty four CMUT linear array structure.

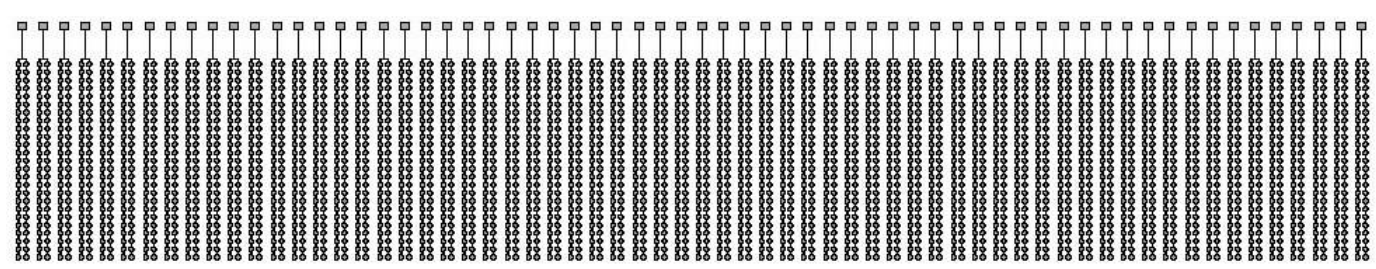

\section{CMUT Array Element and Linear Array Directivity Analysis}

Directivity of an array element or an array refers to a characteristic, namely that the amplitude of its transmitting response or receiving response varies with the azimuth, and is also a kind of attribute in the far field [34-38]. This is usually described or evaluated by using the directivity function, directivity diagram, beam width and sidelobe level, and so on. The following analyses assume that array element or array are located on the $\mathrm{x}$ axis, which is in Oxy plane of 3D space coordinate system as shown in Figure 2, and its surface points to the Oxz plane (the plane formed by $x$ axis and $z$ axis).

\subsection{CMUT Array Element Directivity Analysis}

The micro vibration unit as shown in Figure 1 can be taken as a circular piston with radius $r$, and the directivity function of the circular piston in the Oxz plane is $[34,35,38]$ :

$$
D_{c}(\theta)=\left|\frac{2 J_{1}(k r \sin (\theta))}{k r \sin (\theta)}\right|
$$

where $k$ is the wave number, $r$ is the radius, $\theta$ is the angle between the acoustic line and the $z$ axis, $J_{1}(x)$ is the first-order Bessel function.

According to the Bridge product theorem, a CMUT array element can be regarded as a rectangular planar array composed of $25 \times 2$ micro vibration units, and in the Oxz plane its directivity function is:

$$
D_{e 1}(\theta)=\left|\frac{2 J_{1}(k r \sin (\theta))}{k r \sin (\theta)}\right| \times\left|\frac{\sin \left(k M d_{x} \sin (\theta) / 2\right)}{M \sin \left(k d_{x} \sin (\theta) / 2\right)}\right|
$$

where $M$ is the number of columns of micro vibration cells inside the CMUT array element, and in this case it is $2, d_{x}$ is the distance between the center of micro vibration units in the horizontal direction.

In this design, considering that the diameter of micro vibration unit inside of CMUT array element is very small and even smaller than the wavelength $\lambda$, and $k r=2 \pi r / \lambda \approx 0.59<1$. According to the requirements of the acoustic source in the literature [38], a micro vibration unit can be approximately seen as a point acoustic source to calculate the directivity of CMUT array element. Then a CMUT array element is a planar array composed of $25 \times 2$ linear arrays, and in the Oxz plane its directivity function is [34]:

$$
D_{e 2}(\theta)=\left|\frac{\sin \left(k M d_{x} \sin (\theta) / 2\right)}{M \sin \left(k d_{x} \sin (\theta) / 2\right)}\right|
$$


Considering that the distance between two vibration cells inside of the CMUT array element is very small, it can be described as negligible. Then, a CMUT array element can be regarded as a rectangular piston, of which the length is $b$ and the width is $a$. Its directivity function in the Oxz plane is [34]:

$$
D_{e 3}(\theta)=\left|\frac{\sin (k a \sin (\theta) / 2)}{k a \sin (\theta) / 2}\right|
$$

According to Formula (1), in the polar coordinate system the directivity of circular piston micro vibration unit is shown in Figure 3 when $r$ is $290 \mu \mathrm{m}$. From Figure 3, it can be found that the main lobe is very wide in the directivity diagram of micro vibration unit, and almost without directivity.

Figure 3. Directivity diagram of micro vibration unit.

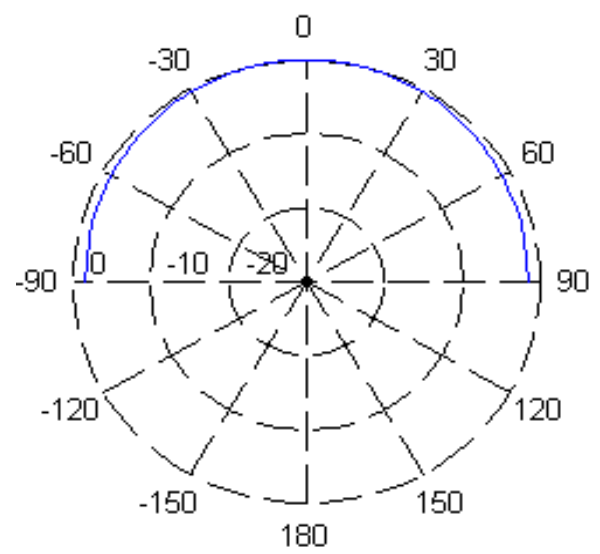

According to Formulas (2)-(4), the directivity of a CMUT array element in the polar coordinate system can be calculated as shown in Figure 4. From Figure 4, it can be seen that the shapes of these three directivity curves acquired according to three methods are quite similar, and according to Formulas (2) and (3), the obtained directivity diagrams almost overlap. Compared with the directivity of micro vibration unit, the directivity of CMUT array element is slightly improved. However, the main lobe in directivity diagram of CMUT array element is still wide no matter which method is adopted to calculate, and its directivity is weak. In order to get stronger directivity, CMUT array elements should be arranged in an array according to certain rules.

Figure 4. Directivity diagram of CMUT array element.

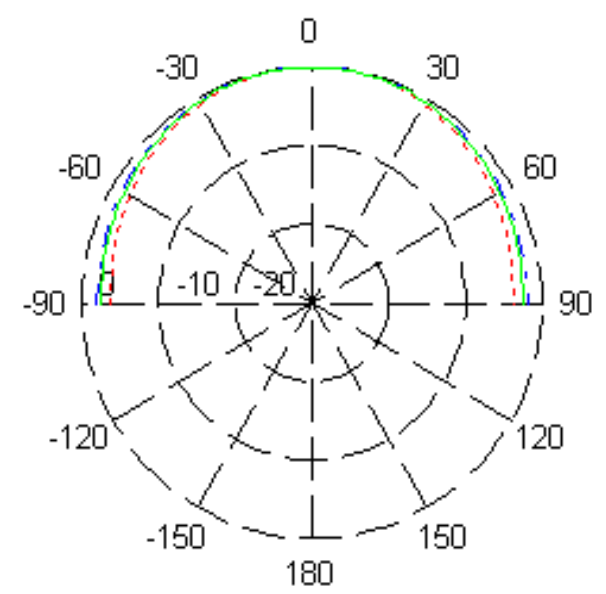




\subsection{CMUT Linear Array Directivity Analysis}

A CMUT array element can follow the Formulas (2)-(4). There are three functions that can be used in order to calculate and analyze its directivity, according to the Bridge product theorem. In addition, the size of the CMUT array element is relatively small and its width is shorter than the wavelength $\lambda$. Moreover, its directivity is weak. A CMUT array element can be approximately seen as a point acoustic source; therefore, another function used to calculate CMUT linear array directivity can be acquired. These four methods are used to calculate and analyze CMUT linear array directivity respectively, and simple comparisons are made between them.

CMUT array element is seen as a rectangular planar array composed of $25 \times 2$ micro vibration units and in the Oxz plane the CMUT linear array directivity function is:

$$
D_{1}(\theta)=\left|\frac{2 J_{1}(k r \sin (\theta))}{k r \sin (\theta)}\right| \times\left|\frac{\sin \left(k M d_{x} \sin (\theta) / 2\right)}{M \sin \left(k d_{x} \sin (\theta) / 2\right)}\right| \times\left|\frac{\sin (k N d \sin (\theta) / 2)}{N \sin (k d \sin (\theta) / 2)}\right|
$$

The CMUT array element is seen as a planar array composed of $25 \times 2$ linear arrays and in the Oxz plane the CMUT linear array directivity function is:

$$
D_{2}(\theta)=\left|\frac{\sin \left(k M d_{x} \sin (\theta) / 2\right)}{M \sin \left(k d_{x} \sin (\theta) / 2\right)}\right| \times\left|\frac{\sin (k N d \sin (\theta) / 2)}{N \sin (k d \sin (\theta) / 2)}\right|
$$

The CMUT array element is seen as a rectangular piston, and in the Oxz plane the CMUT linear array directivity function is:

$$
D_{3}(\theta)=\left|\frac{\sin (k a \sin (\theta) / 2)}{k a \sin (\theta) / 2}\right| \times\left|\frac{\sin (k N d \sin (\theta) / 2)}{N \sin (k d \sin (\theta) / 2)}\right|
$$

The CMUT array element is approximately seen as a point acoustic source, and in the Oxz plane the CMUT linear array directivity function is:

$$
D_{4}(\theta)=\left|\frac{\sin (k N d \sin (\theta) / 2)}{N \sin (k d \sin (\theta) / 2)}\right|
$$

In the Formulas (5)-(8), $N$ is the number of array elements in CMUT linear array, and in this case $N$ is $64, d$ is the distance between the center of array elements in CMUT linear array, and it is $\lambda / 2$ in this case.

Through calculation, the directivity of CMUT linear array can be obtained in Cartesian coordinate system and polar coordinate system, as is shown in Figure 5. Figure 6 shows comparisons of these four directivities. As can be seen from the figure, calculated by the above four methods, the main lobe width of these beam directivity diagrams is almost the same. Within the range from $0 \mathrm{~dB}$ to $30 \mathrm{~dB}$, the sidelobe is consistent, and within the range from $30 \mathrm{~dB}$ to $60 \mathrm{~dB}$ the resulting sidelobe of directivity diagrams obtained by the Formulas (5)-(7) is similar, and it is slightly different compared with the sidelobe of directivity diagram obtained by Formula (8). In addition, it can be seen that the CMUT array element can approximately be seen as a point acoustic source to analyze CMUT linear array directivity without considering the impact of the length of CMUT array element. However, there still exists a sidelobe in the directivity diagram no matter which calculation method is used to calculate. In order to further improve the imaging accuracy and resolution, measures should be taken to reduce the sidelobe. 
Figure 5. CMUT Linear Array Directivity Diagram: (a) directivity diagram calculated by Formula (5); (b) directivity diagram calculated by Formula (6); (c) directivity diagram calculated by Formula (7); and (d) directivity diagram calculated by Formula (8).

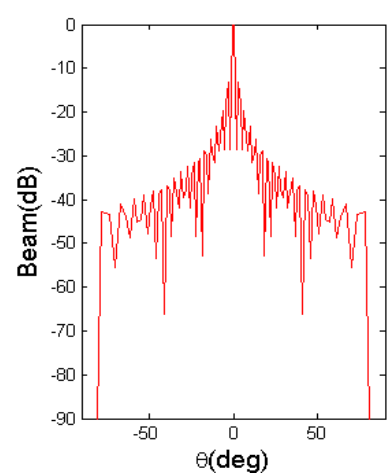

(a)

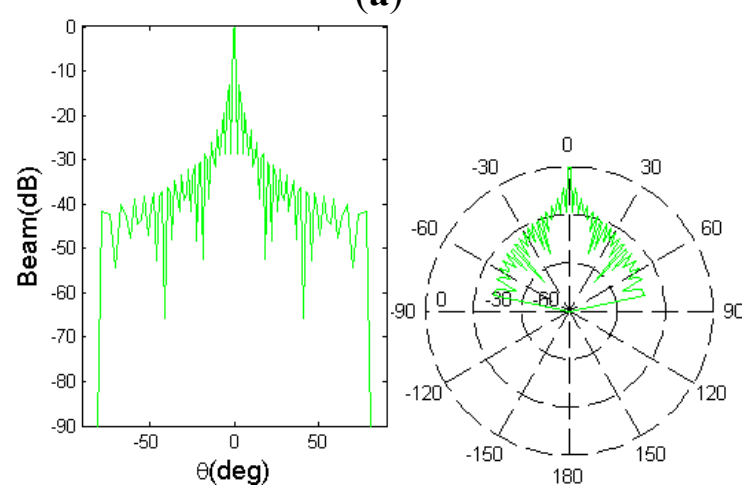

(c)

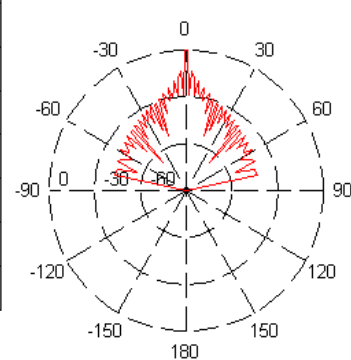

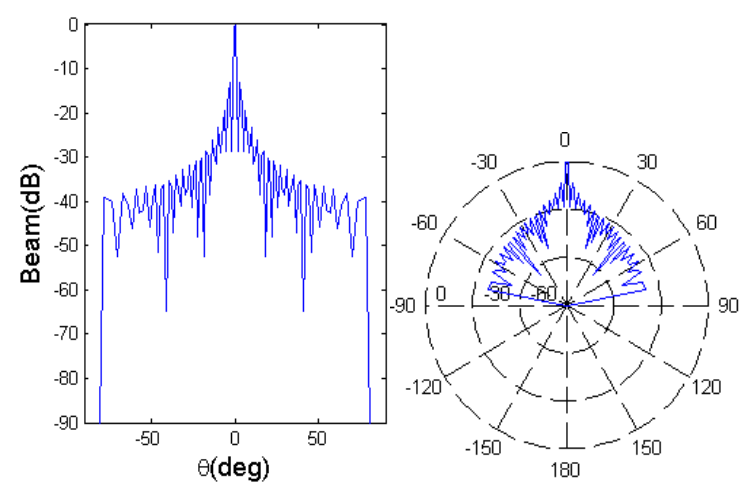

(b)

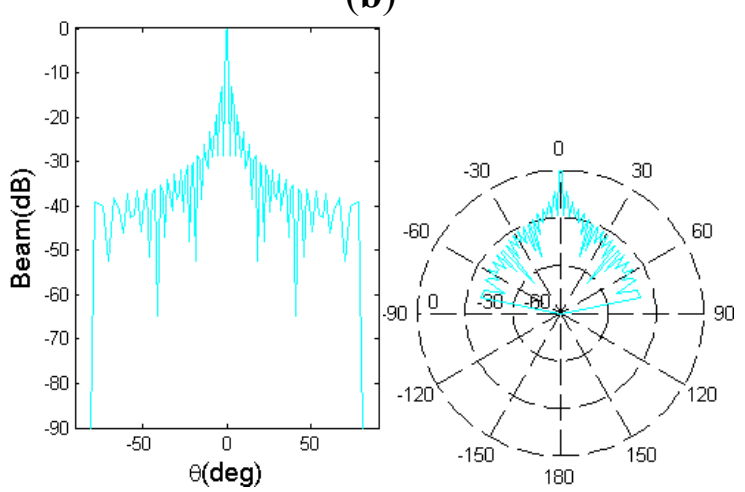

(d)

Figure 6. Directivity comparison.

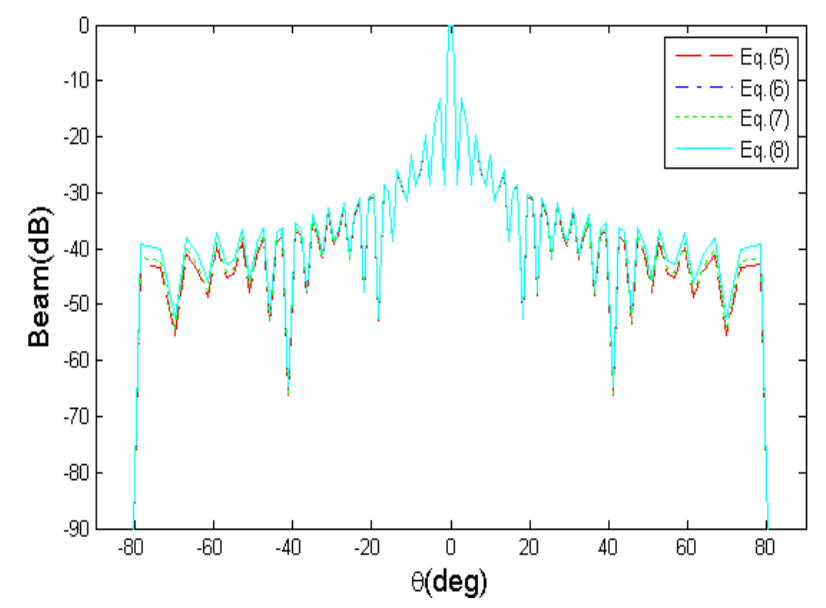

\section{CMUT Linear Array Sidelobe Reduction Method and Implementation}

There are many ways to achieve sidelobe suppression, and the weighting process is one of the most common and effective methods [39]. This paper adopts the Dolph-Chebyshev weighting method and the Taylor weighting method to carry out $-40 \mathrm{~dB}$ sidelobe suppression process respectively, and directivity after process is shown in Figure 7. As can be seen from the figure, the weighting process not only 
reduces the sidelobe compared with the directivity diagram without weighting process, but also the main lobe width remains constant, where the effect of the Taylor weighting method is particularly evident.

Figure 7. CMUT linear array directivity diagram after weighted treatment.

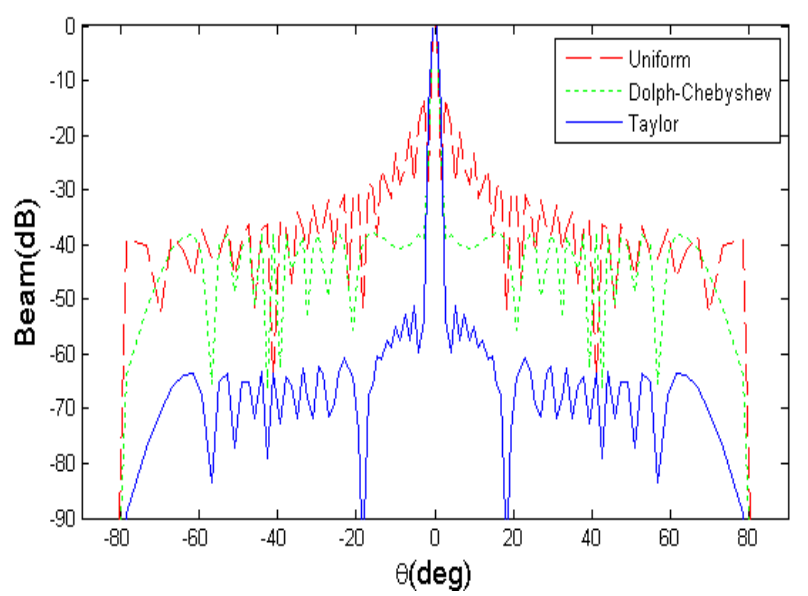

\section{Conclusions}

This paper adopts an integrated vibration membrane structure to design a type of CMUT composed of multiple micro vibration units, and finds that a micro vibration unit almost has no directivity through the directivity analysis. Moreover, the directivity of a single CMUT array element is very weak, so it should be composed into linear array to enhance the directivity. This paper has designed a linear array composed of 64 CMUT array elements and uses a variety of methods to analyze its directivity. Then, through the analysis, it finds that the directivity of designed CMUT linear array is quite strong. In order to reduce the sidelobe of CMUT linear array, the Dolph-Chebyshev weighting method and the Taylor weighting method are used to suppress the sidelobe effectively, and the imaging quality and resolution of the imaging system can be improved further.

\section{Acknowledgments}

The authors wish to thank the Special Fund of the National Natural Science Foundation for project support, funded project (61127008). And this work is supported in part by the National 863 Program (NO.2011AA040404).

\section{Author Contributions}

All work with relation to this paper has been accomplished by all authors' efforts. The idea was proposed by Hongliang Wang. Analysis of the CMUT array was performed by Hongliang Wang and Xiangjun Wang. The CMUT structure was designed by Changde He. The design of the CMUT array was completed with the help from Chenyang Xue.

\section{Conflicts of Interests}

The authors declare that there is no conflict of interest. 


\section{References}

1. Jia, B.X.; Bian, W.F.; Zhao, W.S.; Wang, Z.L. Application and development of piezoelectric ultrasonic transducers. Piezoelectrics Acoustooptics 2004, 27, 131-135. (In Chinese)

2. Wen, J.Q.; Liu, M.L. PZT thick film and high frequency ultrasonic transducer. Tech. Acoust. 2010, 29, 74-80. (In Chinese)

3. Wang, C.; Wang, Z.; Ren, T.L.; Zhu, Y.; Yang, Y.; Wu, X.; Liu, L. A micromachined piezoelectric ultrasonic transducer operating in $\mathrm{d}_{33}$ mode using square interdigital electrodes. IEEE Sens. J. 2007, 7, 967-976.

4. Wang, Z.; Wang, C.; Liu, L. Design and analysis of a PZT-based micromachined acoustic transducer with increased sensitivity. IEEE Trans. Ultrason. Ferroelectr. Freq. Control 2005, 52, 1840-1850.

5. Muralt, P.; Ledermann, N.; Baborowski, J.; Barzegar, A.; Gentil, S.; Belgacem, B.; Petitgrand, S.; Bosseboeuf, A.; Setter, N. Piezoelectric micromachined transducers based on PZT thin films. IEEE Trans. Ultrason. Ferroelectr. Freq. Control 2005, 52, 2276-2288.

6. Muralt, P.; Baborowski, J. Micromachined ultrasonic transducers and acoustic transducers based on piezoelectric thin films. J. Electroceramics 2004, 12, 101-108.

7. Wang, Z.; Zhu, W.; Zhu, H.; Miao, J.; Chao, C.; Zhao, C.; Tan, O.K. Fabrication and characterization of piezoelectric micromachined ultrasonic transducers with thick composite PZT films. IEEE Trans. Ultrason. Ferroelectr. Freq. Control 2005, 52, 2289-2297.

8. Wang, Z.H.; Zhu, W.G.; Miao, J.M.; Zhu, H.; Chao, C.; Tan, O.K. Micromachined Thick Film Piezoelectric Ultrasonic Transducer Array. In Proceedings of the 13th Intemational Conference on Solid-State Sensors, Actuators and Microsystems, Seoul, Korea, 5-9 June 2005; pp. 883-886.

9. Eccardt, P.C.; Niederer, K.; Scheiter, T.; Hierold, C. Surface Micromachined Ultrasound Transducers in CMOS Technology. In Proceedings of the 1996 IEEE Ultrasonic Symposium, San Antonio, TE, USA, 3-6 November 1996; pp. 959-962.

10. Eccardt, P.C.; Niederer, K.; Fischer, B. Micromachined Transducers for Ultrasonic Applications. In Proceedings of the 1997 IEEE Ultrasonic Symposium, Toronto, Canada, 5-8 October 1997; pp. 1609-1618.

11. Mills, D.M.; Smith, L.S. Real Time in-vivo Imaging with Capacitive Micromachined Ultrasonic Transducer (cMUT) Linear Arrays. In Proceedings of the 2003 IEEE Ultrasonic Symposium, Honolulu, HA, USA, 5-8 October 2003; pp. 568-571.

12. Oralkan, Ö.; Ergun, A.S.; Johnson, J.A.; Karaman, M.; Demirci, U.; Kaviani, K.; Khuri-Yakub, B.T. Capacitive micromachined ultrasonic transducers: Next-generation arrays for acoustic imaging? IEEE Trans. Ultrason. Ferroelectr. Freq. Control 2002, 49, 1596-1610.

13. Ladabaum, I.; Jin, X.; Soh, H.T.; Atalar, A.; Khuri-Yakub, B.T. Surface micromachined capacitive ultrasonic transducers. IEEE Trans. Ultrason. Ferroelectr. Freq. Control 1998, 45, 678-690.

14. Ergun, A.S.; Yaralioslu, G.G.; Khuri-Yakub, B.T. Capacitive micromachined ultrasonic Transducers: Theory and technology. J. Aerosp. Eng. 2003, 16, 76-84.

15. Suzuki, K.; Higuchi, K.; Tanigawa, H. A silicon electrostatic ultrasonic transducer. IEEE Trans. Ultrason. Ferroelectr. Freq. Control 1989, 36, 620-627. 
16. Haller, M.I.; Khuri-Yakub, B.T. A surface micromachined electrostatic ultrasonic air transducer. IEEE Trans. Ultrason. Ferroelectr. Freq. Control 1996, 43, 1-6.

17. Hansen, S.T.; Mossawir, B.J.; Sanli Ergun, A.; Degertekin, F.; Khuri-Yakub, B.T. Air-Coupled Nondestructive Evaluation Using Micromachined Ultrasonic Transducers. In Proceedings of the 1999 IEEE Ultrasonics Symposium, Lake Tahoe, NV, USA, 17-20 October 1999; pp. 1037-1040.

18. Johnson, J.; Oralkan, Ö.; Demirci, U.; Ergun, S.; Karaman, M.; Khuri-Yakub, P. Medical imaging using capacitive micromachined ultrasonic transducer arrays. Ultrason. 2002, 40, 471-476.

19. Oralkan, O.; Ergun, A.S.; Cheng, C.H.; Johnson, J.A.; Karaman, M.; Lee, T.H.; Khuri-Yakub, B.T. Volumetric Imaging Using 2D Capacitive Micromachined Ultrasonic Transducer Arrays (CMUTs): Initial results. In Proceedings of the 2002 IEEE Ultrasonics Symposium, Munich, Germany, 8-11 October 2002; pp. 1083-1086.

20. Zhuang, X.; Ergun, A.S.; Huang, Y.; Wygant, I.O.; Oralkan, O.; Khuri-Yakub, B.T. Integration of trench-isolated through-wafer interconnects with 2D capacitive micromachined ultrasonic transducer arrays. Sens. Actuators A Phys. 2007, 138, 221-229.

21. Zhuang, X.; Lin, D.S.; Oralkan, Ö.; Khuri-Yakub, B.T. Fabrication of flexible transducer arrays with through-wafer electrical interconnects based on trench refilling with PDMS. J. Microelectromech. Syst. 2008, 17, 446-452.

22. Wygant, I.O.; Jamal, N.; Lee, H.; Nikoozadeh, A.; Oralkan, O.; Karaman, M.; Khuri-Yakub, B. An integrated circuit with transmit beam forming flip-chip bonded to a 2-D CMUT array for 3-D ultrasound imaging. IEEE Trans. Ultrason. Ferroelectr. Freq. Control 2009, 56, 2145-2156.

23. Choe, J.W.; Oralkan, O.; Nikoozadeh, A.; Bhuyan, A.; Lee, B.C.; Gence, M.; Khuri-Yakub, B.T Real-Time Volumetric Imaging System for CMUT Arrays. In Proceedings of the 2011 IEEE Ultrasonics Symposium, Orlando, FL, USA, 18-21 October 2011.

24. Bhuyan, A.; Choe, J.W.; Lee, B.C.; Wygant, I.; Nikoozadeh, A.; Oralkan, O.; Khuri-Yakub, B.T. $3 \mathrm{D}$ Volumetric Ultrasound Imaging with a $32 \times 32$ CMUT Array Integrated with Front-End ICs Using Flip-Chip Bonding Technology. In Proceedings of the Solid-State Circuits Conference Digest of Technical Papers (ISSCC), 2013 IEEE International, San Francisco, CA, USA, 17-21 February 2013; pp. 396-397.

25. Kaviani, K.; Oralkan, O.; Khuri-Yakub, P.; Wooley, B.A. A multichannel pipeline analog-to-digital converter for an integrated 3-D ultrasound imaging system. IEEE J. Solid State Circuits 2003, 38, 1266-1270.

26. Wygant, I.O.; Karaman, M.; Oralkan, Ö.; Khuri-Yakub, B.T. Beamforming and Hardware Design for a Multichannel Front-End Integrated Circuit for Real-Time 3-D Catheter-Based Ultrasonic Imaging. In Proceedings of the SPIE 6147, Medical Imaging 2006: Ultrasonic Imaging and Signal, San Diego, CA, USA, 11 February 2006.

27. Oralkan, Ö.S.; Hansen, T.; Bayram, B.; Yaralioglu, G.G.; Ergun, A.S.; Khuri-Yakub, B.T. High-Frequency CMUT Arrays for High-Resolution Medical Imaging. In Proceedings of the 2007 IEEE Ultrasonics Symposium, Montréal, Canada, 24-27 August 2004; pp. 399-402.

28. Zhuang, X.; Wygant, I.O.; Lin, D.S.; Kupnik, M.; Oralkan, Ö.; Khuri-Yakub, B.T. Trench-Isolated CMUT Arrays with a Supporting Frame: Characterization and Imaging Results. In Proceedings of the 2007 IEEE Ultrasonics Symposium, New York, NY, USA, 28-31 October 2007; pp. 507-510. 
29. Caronti, A.; Caliano, G.; Carotenuto, R.; Savoia, A.; Pappalardo, M.; Cianci, E.; Foglietti, V. Capacitive micromachined ultrasonic transducer (CMUT) arrays for medical imaging. Microelectron. J. 2006, 37, 770-777.

30. Song, G.D.; Liu, J.; Li, D.C. Structure design and simulation of capacitive microfabricated ultrasonic transducer. Nanotechnol. Precis. Eng. 2006, 5, 156-159. (In Chinese)

31. Tian, J.; Wang, C.H.; Xu, L.; Qiao, D.H.; Ma, J.; Hao, Z.H.; Wei, J.H. Silicon condenser microphone with round membranes. Chin. J. Sens. Actuators 2006, 12, 2297-2299. (In Chinese)

32. Hao, Z.H.; Tang, L.; Qiao, D.H. Analysis of the resonant frequency of capacitive micro-machined ultrasonic transducer (cMUT) with Finite Element Method. Tech. Acoust. 2009, 28, 133-134. (In Chinese)

33. Miao, J.; He, C.D.; Lian, D.Q.; Zhang, H.; Yu, J.Q.; Wan, K.J.; Xue, C.Y.; Zhang, W.D. Design of MEMS capacitiVe ultrasonic transducer based on wafer bonding technology. Chin. J. Sens. Actuators 2012, 25, 1653-1658. (In Chinese)

34. Luan, G.D.; Zhang, J.D.; Wang, R.Q. Piezoelectric Transducer and Transducer Array; Peking University Press: Beijing, China, 2005; pp. 326-435. (In Chinese)

35. Kino, G.S. Acoustic Waves: Devices, Imaging, and Analog Signal Processing; Prentice-Hall: Englewood Cliffs, NJ, USA, 1987.

36. Kinsler, L.E.; Frey, A.R.; Coppens, A.B.; Sanders, J.V. Fundamentals of Acoustics, 4th ed.; Wiley: New York, NY, USA, 2000.

37. Angelsen, B.A.J. Ultrasound Imaging: Waves, Signals, and Signal Processing; Emantec: Trondhejm, Norway, 2000.

38. Du, G.H.; Zhu, Z.M.; Gong, X.F. Acoustics Foundation, 3rd ed.; Nanjing University Press: Nanjing, China, 2012; pp. 222-223. (In Chinese)

39. Van Trees, H.L. Optimum Array Processing; Wiley: New York, NY, USA, 2002.

(C) 2014 by the authors; licensee MDPI, Basel, Switzerland. This article is an open access article distributed under the terms and conditions of the Creative Commons Attribution license (http://creativecommons.org/licenses/by/3.0/). 